\begin{tabular}{|c|c|c|}
\hline $\begin{array}{l}\text { PKS } \\
\text { PULII } \\
\text { KNDOWLEDGE } \\
\text { KROJECT }\end{array}$ & $\begin{array}{c}\text { REVISTA DE GEOGRAFIA } \\
\text { (RECIFE) } \\
\text { http://www.revista.uffe.br/revistageografia }\end{array}$ & $\begin{array}{l}\text { OJS } \\
\text { OPEN } \\
\text { JOUNAL } \\
\text { SYSTEM }\end{array}$ \\
\hline
\end{tabular}

\title{
LA "RE-EXISTENCIA" DESDE LOS TERRITORIOS COMUNITARIOS Y EL PATRIMONIO BIOCULTURAL FRENTE A LA MEGA-MINERÍA A CIELO ABIERTO EN MÉXICO
}

\author{
Letizia Silva Ontiveros $^{1}$, Luciano Concheiro Bórquez ${ }^{2}$
}

\begin{abstract}
${ }^{1}$ Doctora en Geografía, UNAM. Postdoctorante del Instituto de Geografía, Universidad Nacional Autónoma de México.E-mail: lsilva@igg.unam.mx

${ }^{2}$ Doctor en Desarrollo Rural, UAM-X. Investigador del Departamento de Producción Económica y docente del Posgrado en Desarrollo Rural de la Universidad Autónoma Metropolitana - Xochimilco, México. E-mail: concheir@gmail.com
\end{abstract}

Artigo recebido em 30/12/2017 e aceito em 18/03/2018

\begin{abstract}
RESUMEN
La problemática social y ambiental derivada del nuevo extractivismo minero va en aumento, para enfrentarla se han generado procesos de resistencia y lucha a nivel local y regional que han integrado temas de defensa del patrimonio histórico, planeación regional, proyectos en base a derechos humanos y ambientales, recuperación y re-existencia de modos de vida campesinos, entre otros. En los múltiples casos donde se ha impuesto esta megaminería devastadora se ha puesto a la orden del día la necesidad de un enfoque de recuperación, no sólo en el aspecto físico-material y de restauración de suelos de los sitios mineros, sino en una perspectiva que parta de los procesos históricos tanto comunitarios como regionales, sus formas de sobrevivencia y lucha para regenerar la propia vida de los sitios y restaurar el tejido social.

Palabras-clave: Minería a cielo abierto, Territorios de re-existencia, patrimonio biocultural.
\end{abstract}

\section{THE "RE-EXISTENCE" FROM THE COMMUNITY TERRITORIES AND THE BIOCULTURAL HERITAGE AGAINST MEGA OPEN PIT MINING IN MEXICO}

\begin{abstract}
The social and environmental problems arising from the new mining extractivism is increasing, to face it have been processes of resistance and struggle at local and regional level that have integrated issues of defense of historical heritage, regional planning, projects based on human and environmental rights, recovery and reexistence of peasant ways of life, among others. In the multiple cases where this devastating mega-mining has been imposed, the need for a recovery approach has been placed on the agenda, not only in the physical-material aspect and soil restoration of the mining sites, but in a perspective that starts from historical processes both community and regional, their survival and struggle to regenerate the lives of the sites and restore the social fabric.
\end{abstract}

Keywords: Open pit mining, Re-existence territories, Biocultural heritage.

\section{INTRODUCCIÓN}

Este escrito se centra en la identificación del patrimonio biocultural como un elemento que las comunidades utilizan en sus luchas cuando ya han sido afectadas por la actividad de la minería a cielo abierto. Ese tipo de patrimonio, socialmente se percibe en riesgo, y se 
construyen agendas y sobre todo prácticas que, desde las propias tradiciones locales y las epistemologías locales, son utilizadas para establecer territorios de re-existencia.

La finalidad de este trabajo, es en primera instancia, dar cuenta de la problemática social y ambiental que ha generado en México el vertiginoso despliegue de la minería a cielo abierto ligada con otros mega-proyectos, en particular las presas, que han sido denominados en su conjunto, como "proyectos de muerte". Ligado a lo anterior, presentamos cómo las propias comunidades que se encuentran en riesgo de desaparición, establecen redes sociales para reconstruir sus territorios sobre la base de su patrimonio biocultural, al abarcar formas de vivir y organizarse para una re-existencia, como forma continua, cotidiana e inventiva de lucha. Este trabajo analiza experiencias sociales de espacios que ya han sido devastados por la mega-minería, como en el caso de Cerro de San Pedro en el estado de San Luis Potosí en México, para recuperar el sentido de sus territorios, reconfigurando y re-semantizando lo perdido, y que han hecho un intento por destacar el patrimonio histórico y biocultural de los pueblos y comunidades. De esta manera se pretende recuperar la visión de que quienes son los encargados del resguardo de los bienes naturales y culturales que no solo comprende a las poblaciones indígenas, sino las que se definen como mestizas y que legalmente se definen como "comunidades locales y equiparables"1 Desde esta visión, se analiza cómo la población afectada, retoma sus prácticas productivas, sus conocimientos tradicionales, construye nuevos tipos de defensa referidos al paisaje, a rutas e itinerarios, establece geopatrimonios y define en sus prácticas, lo que pueden llamarse:“territorios de re-existencia”.

\section{MINERÍA COLONIAL Y NEOCOLONIAL}

La minería en México es una actividad que, desde su surgimiento en la época colonial, tiene como rasgo ser un modelo dependiente del exterior y de acaparar grandes extensiones de tierras. Desde el siglo XVI, los conquistadores obtuvieron así grandes territorios originalmente ocupados por los pueblos originarios, despojando y explotando primero a los indígenas y después a los esclavos traídos de África. Dicha explotación fue desmedida, no sólo porque generó una catástrofe demográfica, sino porque el modelo de apropiación del espacio constituyó la destrucción de las formas indígenas de organización social, así como la erosión de sus valores culturales (Silva, 2014: 49). Actualmente, en la nueva etapa de la

\footnotetext{
${ }^{1}$ Ver Eckart Boege (2015), "Patrimonio biocultural y los derechos culturales de los pueblos indígenas, comunidades locales y equiparables"; pp. 1.
} 
mega-minería el colonialismo se ha renovado en una suerte de neocolonialismo, imponiendo actualizadas formas de acumulación por despojo.

Por su intensidad y dimensión espacial la actividad minera representa en México el principal proceso de acumulación de capital. La llegada de nuevas mega-mineras en países como México, ha tenido un fuerte impacto en la población tanto por las implicaciones ambientales, como por la desaparición de poblados y alteraciones del paisaje a gran escala. Es precisamente la utilización del prefijo "mega", el marco de referencia para describir las dimensiones de una nueva modalidad de una actividad que, sin embargo, es bastante conocida y por demás antigua. El prefijo revela mayores dimensiones, un número cuantioso de proyectos, mayor magnitud de recursos y trabajo, y la ocupación e impacto territorial de manera más intensiva y extensiva que la minería tradicional. Esta es la amenaza hecha realidad, pues como menciona Héctor Alimonda (2011: 12): "para comienzos del siglo XXI (pasados quinientos años de la minería colonial), el interés renovado por la apropiación y explotación intensiva de los recursos minerales de los países latinoamericanos, se expresa en la multiplicación de mega-proyectos de extracción en todo el continente".

De acuerdo con el Informe de la Dirección General de Minas (2015), la mayor parte de la inversión está destinada a la minería que llevan a cabo empresas que explotan oro y plata, realizada básicamente por empresas canadienses, que cuentan en México con el $68.8 \%$ de empresas mineras y el 65\% de inversión (véase Figura 1).

Figura 1. Proporción de empresas con proyectos mineros en México.
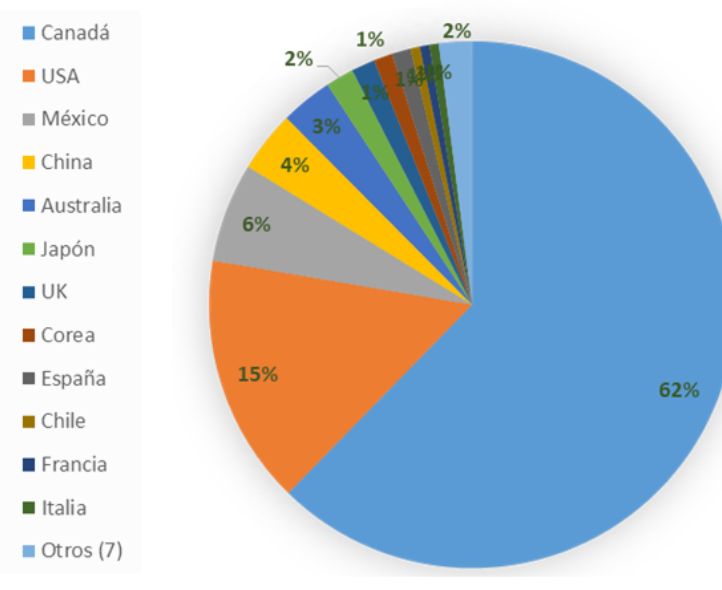

\begin{tabular}{|c|c|}
\hline $\begin{array}{c}\text { Nacionalidad de } \\
\text { empresas }\end{array}$ & $\begin{array}{l}\text { Núm de } \\
\text { empresas }\end{array}$ \\
\hline Canadá & 215 \\
\hline USA & 53 \\
\hline México & 21 \\
\hline China & 13 \\
\hline Australia & 11 \\
\hline Japón & 6 \\
\hline UK & 5 \\
\hline Corea & 4 \\
\hline España & 4 \\
\hline Chile & 2 \\
\hline Francia & 2 \\
\hline Italia & 2 \\
\hline Argentina - Italia & 1 \\
\hline Bélgica & 1 \\
\hline Filipinas & 1 \\
\hline India & 1 \\
\hline Irlanda - UK & 1 \\
\hline Perú & 1 \\
\hline Suiza & 1 \\
\hline Total & 345 \\
\hline
\end{tabular}

Elaboración propia con información de Secretaría de Economía, Servicio geológico Mexicano. 
Esto nos habla de una transnacionalización a nivel global de la actividad minera y, por su carácter especulativo, del proceso de financiarización de la economía mundial, por eso, a pesar de haber intensificado la explotación y beneficio de minerales, el rubro más importante de la inversión es en la exploración (Silva, 2014: 201). En poco más de una década, de 2006 a 2017, México cuenta con el 6\% de la inversión a nivel mundial y ocupa el cuarto lugar sólo después de Canadá, Australia y Estados Unidos².

Figura 2. Porcentaje de inversión minera en el mundo.

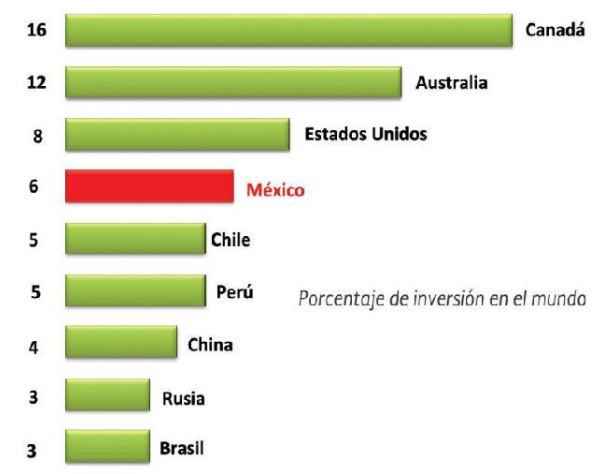

Fuente: Metal Economics Group 2013

Las políticas neoliberales en torno a la minería son en México particularmente permisivas e impulsan la inversión extranjera con nuevos desarrollos. En el Artículo sexto de la Ley Minera, en el que se otorga preferencia a esta actividad sobre cualquier otro uso, dice con claridad absoluta: “... la exploración, explotación y beneficio de los minerales o sustancias a que se refiere esta Ley son de utilidad pública, serán preferentes sobre cualquier otro uso o aprovechamiento del terreno, con sujeción a las condiciones que establece la misma, y únicamente por Ley de carácter federal podrán establecerse contribuciones que graven estas actividades". En estas condiciones, es difícil, si no imposible, discutir el futuro minero de una comunidad, de una zona o del país mismo, cuando la posición pública del gobierno, formulada en reiteradas ocasiones, es abiertamente favorable a la minería a gran escala. Declarar a priori que la minería "va porque va", desactiva las posibilidades para diálogos francos y abiertos (Acosta 2009:125). Una vez cubiertos los laxos requisitos de ley, el gobierno ha procedido a tramitar las concesiones de los lotes mineros donde se

${ }^{2}$ Metals Economics Group (2012). 
desarrollarán los proyectos, obteniendo las empresas la cesión de concesiones mineras por 50 años prorrogables a un segundo periodo, es decir, otros 50 años más ${ }^{3}$.

Producto de las políticas neoliberales y de los cambios a la legislación mexicana, en cualquier fase de la actividad extractiva, las empresas mineras tienen en su poder la toma de decisiones en los espacios que les han sido concesionados. Son las transnacionales y en general los monopolios mineros, los que tienen prioridad para establecen un dominio territorial al ser la minería en México una "actividad prioritaria y de utilidad pública", frente a cualquier otro tipo de uso, aunque sean áreas sensibles o naturales protegidas. En el caso mexicano las concesiones mineras abarcan cerca de la mitad de todo el territorio nacional (Figura 3). Estas concesiones implican directamente la posesión de las mineras sobre los territorios campesinos en propiedad social (ejidos y comunidades que representan el $54 \%$ del territorio nacional) y aún sobre las propiedades privadas y la imposición un modo externo de organización del espacio, sin tomar en cuenta los proyectos de vida y actividades basadas en los conocimientos y memorias colectivas existentes a nivel local o regional.

La tecnología extractiva de las denominadas explotaciones en "tajos a cielo abierto", se inserta perfectamente para llevar a cabo la explotación, ya que básicamente consiste en la voladura de material rocoso para ser transportado y tratado con grandes volúmenes de agua combinadas con ácido sulfúrico, mercurio y/o cianuro (según se trate de los minerales a extraer) a fin de ser separados del material estéril. Este tipo de tecnología exige destruir enormes extensiones de superficie rocosa, en ocasiones en áreas montañosas, la aplicación de grandes cantidades de explosivos y de sustancias tóxicas y el uso intensivo de dos insumos clave: agua y energía.

\footnotetext{
${ }^{3}$ Publicado en el Diario Oficial de la Federación (DOF), 26 de junio de 1992, artículo 19, frac. VII.
} 
Revista de Geografia (Recife) V. 35, No. 2, 2018

Figura 3. Concesiones mineras en México.

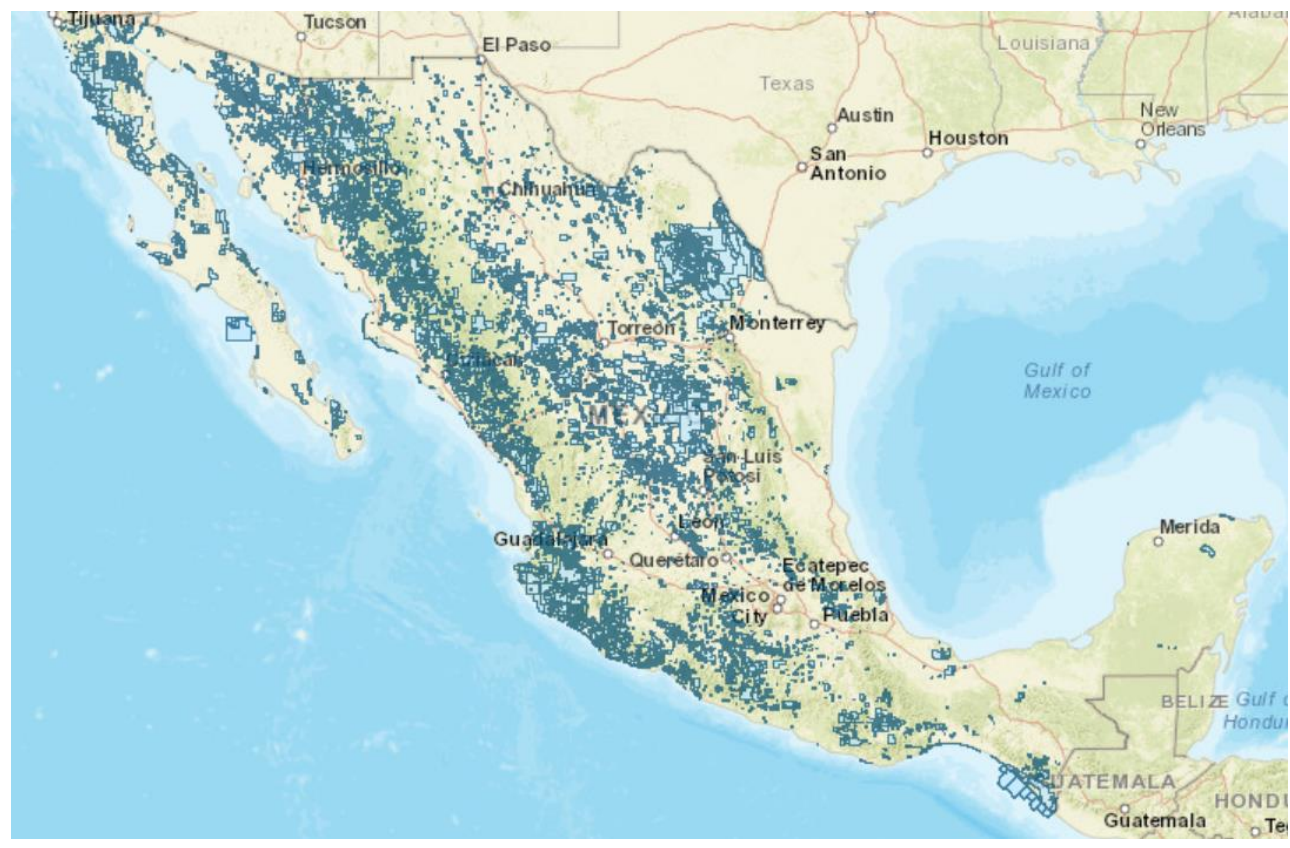

Fuente: Cartografía del Sistema de Administración Minera, Secretaría de Economía, 2017

En la Figura 4 se muestra el volumen de agua concesionado a empresas mineras, tomando en cuenta que casi dos terceras partes de México (del centro al norte) son semidesérticas y desérticas.

Figura 4. Volumen de agua concesionado a mineras.

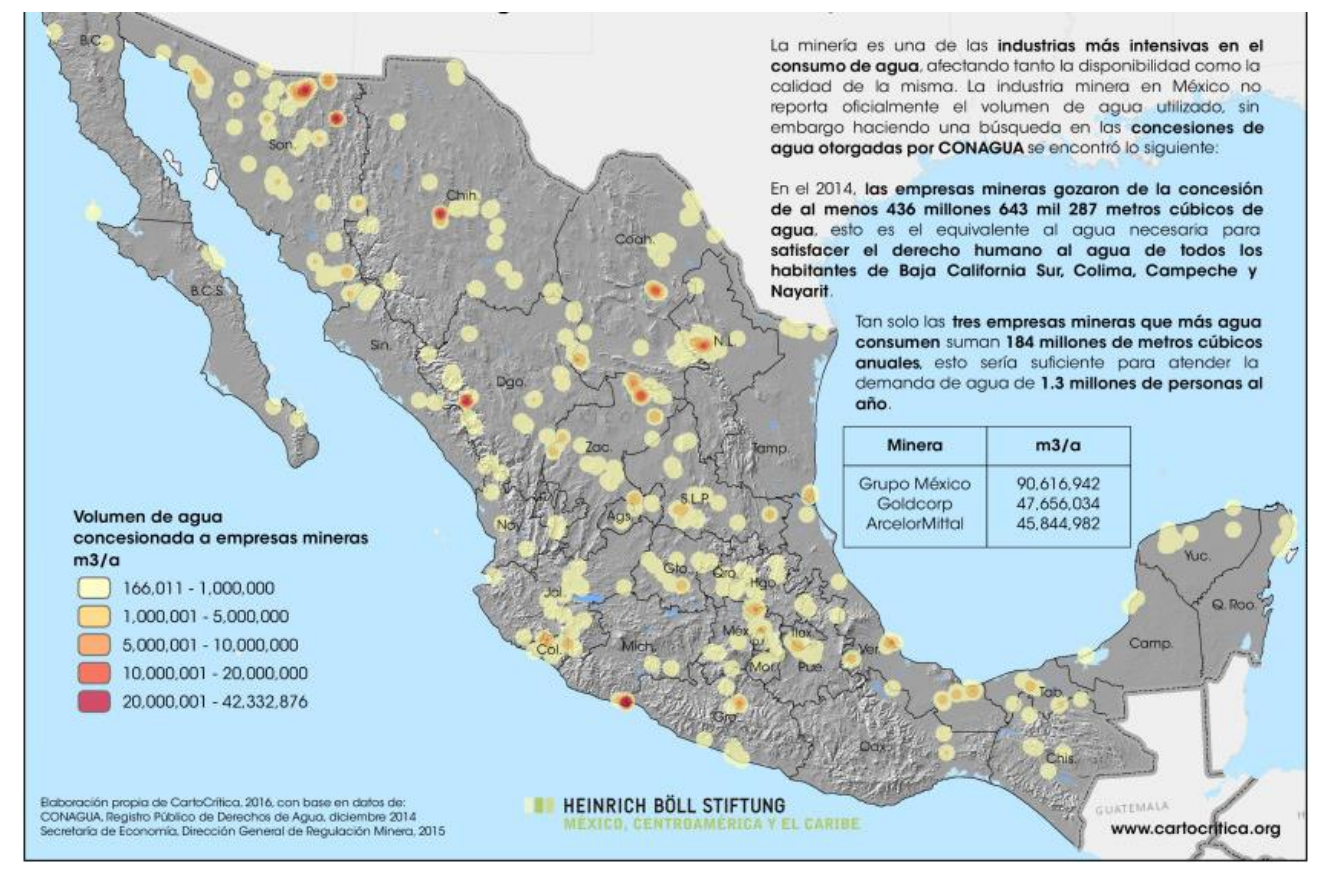

Fuente: www.cartocritica.org 
Maristella Svampa (2011:173) señala que la diferencia con respecto a la minería tradicional, es que implica niveles aún mayores de afectación ambientales, generando cuantiosos pasivos, en ocasiones irreversibles, al tiempo que requiere un uso desmesurado de recursos naturales como insumos, entre ellos el agua y la energía, ambos imprescindibles para sus operaciones, así como transformar de manera violenta la geografía de los territorios explotados.

Cuando se da a conocer el proyecto de minería por medio de la "Manifestación de Impacto Ambiental”, se señala normalmente que la afectación será irreversible, irremediable e irreparable, y así se legitima que haya sido aceptado. La Ley vigente avala este señalamiento y solo pide que se indique cómo se hará la compensación o mitigación del daño, cuando no sea posible su remediación. Recordemos que la contaminación relacionada con este tipo de minería invade el aire con polvo, combustibles tóxicos, vapores o gases de cianuros, mercurio o dióxido de azufre, además se reduce la presencia de agua subterránea, además de contaminarla, hay eliminación del suelo en el área de explotación y con la supresión de vegetación desaparece la biodiversidad de la zona. Aun así, con todo este panorama desolador, por impactos de la mega minería, el territorio de México está concesionado en gran parte y se anuncia que en los próximos años se acabará de concesionar hasta el 75\%

\section{CONFLICTOS DERIVADOS DE LA MINERÍA}

Debido a la problemática social y ambiental que genera o puede llegar a representar la mega minería, se han desatado conflictos en torno a la negativa de los pueblos y comunidades donde se pretenden establecer las actividades mineras. Es necesario aclarar que la actividad minera no solamente está relacionada con la extracción de minerales, sino que abarca las siguientes fases: a) Prospección (Reconocimiento del área para identificar el mineral), b) Exploración (Verificación-ubicación del mineral, y factibilidad de su extracción), c) Explotación (Procesos para extraer el mineral), d) Beneficio (Limpieza y refinación), y e) Cierre (Trabajos para abandonar el sitio).

La población se ha movilizado en las diferentes fases del proceso minero, por lo que algunos conflictos pueden desarrollarse en las fases iniciales, cuando aún están en proceso los permisos correspondientes, o bien, durante las actividades que actúan directamente en los territorios, lo que incide en que el conflicto se desarrolle de manera distinta. Es así que mientras algunos pueden frenar el proceso desde antes que se lleve a cabo la operación directa, otros aun cuando se movilizan, puede ser que la empresa continúe con sus fases de 
operación y en ese sentido no logren suspender las actividades mineras. De cualquier manera, en ambos casos la incertidumbre continúa al no asegurarse de que la minería sea cancelada, solamente se suspende pero no se anulan las concesiones.

Svampa, menciona que una de las consecuencias del "actuar extractivista" ha sido la explosión de conflictos socioambientales, visibles en la potenciación de las luchas ancestrales por la tierra de la mano de los movimientos indígenas y campesinos, así como en el surgimiento de nuevas formas de movilización y participación ciudadana, centradas en la defensa de los recursos naturales (definidos como "bienes comunes"), la biodiversidad y el ambiente. Así, los conflictos socioambientales son "aquellos ligados al acceso, conservación y control de los recursos naturales, que suponen por parte de los actores enfrentados, intereses y valores divergentes en torno a los mismos, en un contexto de asimetría de poder" (Svampa, 2012: 186).

Asistimos por lo anteriormente dicho, a ciertas transformaciones de los movimientos. En principio se relacionaban con la protección y defensa de un recurso en particular: agua, tierra, vivienda, propiedad común, etc., para dar paso a contemplar el territorio y las formas en que la población se ubica dentro de éste.

Figura 5. Esquema de transformación de luchas en común.

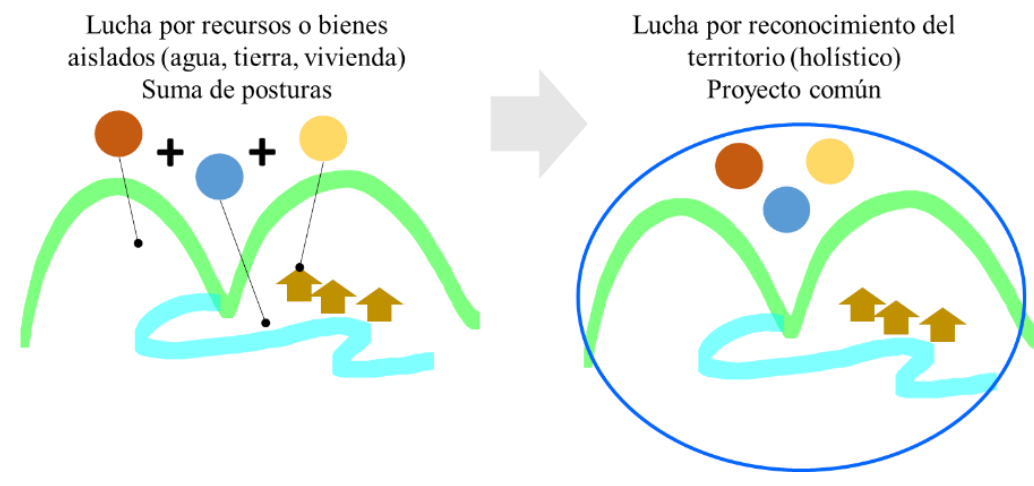

Elaboración propia

De esta manera se contemplaron los temas en conflicto, no como suma de partes, sino como un proyecto holístico, o dicho desde otro ángulo, desde la totalidad y por ello politicidad de las luchas para formar y construir en la práctica un proyecto en común. A partir de esto, se relacionan, en redes y frentes de lucha, además con movimientos que detectaron conflictos similares en otros territorios, identificando un modelo "extractivista", por lo que 
ahora la lucha también es contra la implantación de este tipo de modelo que implica no solo minería, sino otros tipos de mega proyectos (presas, fracking, agroindustria, mega-turismo).

Figura 6. Esquema de luchas por el territorio en defensa ante el modelo extractivo.

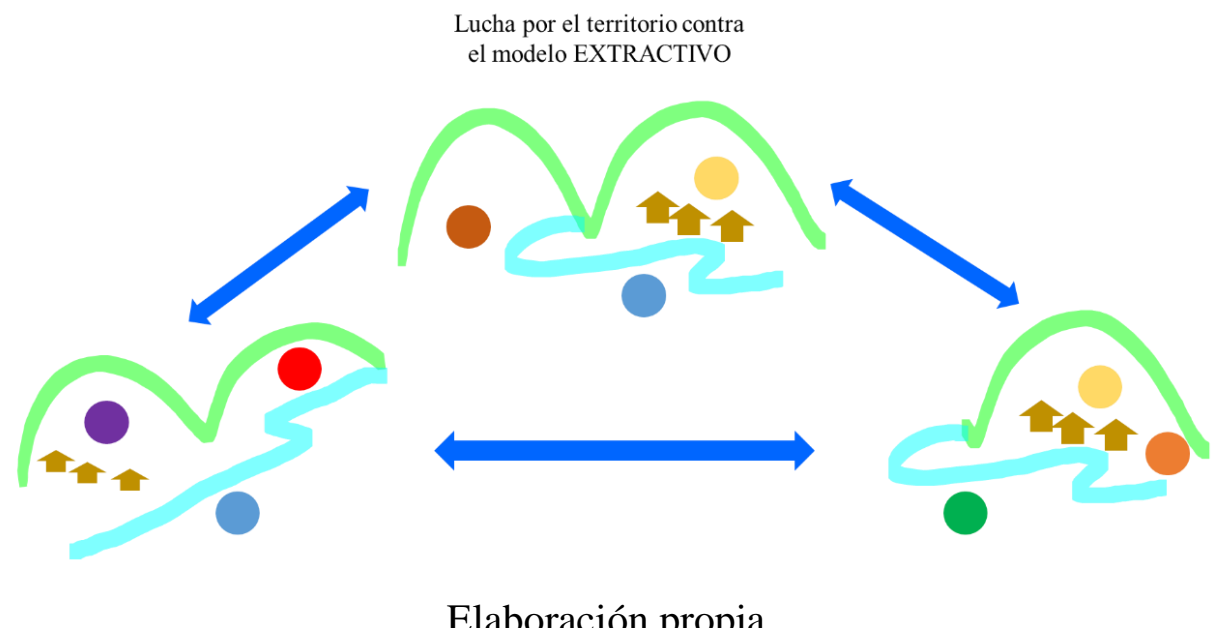

Los datos que se encuentran en los registros de dos de los observatorios sobre conflictos mineros, el Observatorio de Conflictos Mineros en América Latina (OCMAL) y el mapa de Justicia Ambiental del Environmental Justice Organisations, Liabilities and Trade (EJOLT), arrojan para México, de 1999 a 2015, 41 conflictos relacionados con proyectos mineros. De ellos, el 46\% se encuentran en los estados de Chihuahua, Oaxaca y Puebla con cinco conflictos en cada entidad. Le siguen con un 27\%, Baja California Sur, San Luis Potosí y Sonora con tres conflictos cada uno; con un 18\% se encuentran Coahuila, Colima, Jalisco, Michoacán y Zacatecas con dos conflictos por entidad, y con el 9\% restante, los estados de Baja California Norte, Durango, Guanajuato, Guerrero, Hidalgo, Morelos, Querétaro y Veracruz, con un conflicto cada uno.

Figura 7. Proporción de conflictos por estado

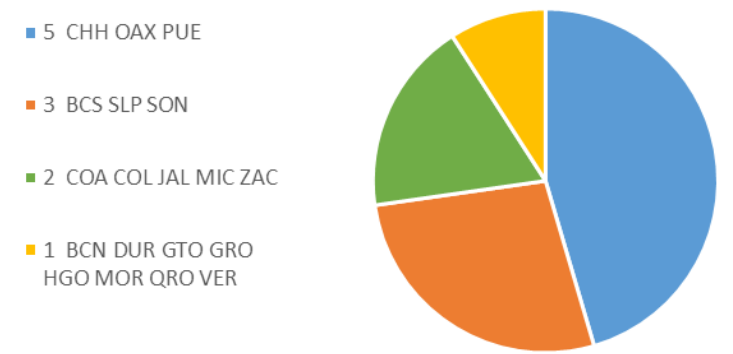

Elaboración propia con base en OCMAL y EJOLT 2017 
Cabe señalar que casi el $70 \%$ de los conflictos detectados están relacionados con minería de metales preciosos (oro y plata) con 28 proyectos, siguiéndoles los que extraen Cobre (17\%) y Magneso y Fierro (12\%). De los conflictos que se relacionan con metales preciosos, el $53 \%$ se encuentran en poblaciones indígenas. En la tabla 1, los conflictos relacionados con las empresas y el tipo de población (verde, indígena; azul, mestiza).

Tabla 1. Conflictos mineros, empresas y minerales que pretenden explotar.

\begin{tabular}{|c|c|c|c|c|}
\hline Ubicación del Conflicto & $\begin{array}{l}\text { Inicio del } \\
\text { Conflicto }\end{array}$ & Empresas & Minerales & Población \\
\hline \multicolumn{5}{|l|}{ OCMAL } \\
\hline $\begin{array}{l}\text { Municipio Santa María de Zaniza, Región Sierra } \\
\text { Sur, Oaxaca }\end{array}$ & 1999 & Altos Hornos de México S.A de C.V & $\mathrm{Fe}$ & \\
\hline Municipalidad de Aquila, Estado de Michoacán, & 2000 & Las Encinas S.A de C.V, Ternium & $\mathrm{Fe}$ & \\
\hline Municipio de Ocotlan, Oaxaca & 2002 & Continuum Resources, Minera Natividad & $\mathrm{Au} \mathrm{Ag}$ & \\
\hline Motozintla, Chiapas & 2007 & Linear Gold Corp & $\mathrm{Au}$ & \\
\hline San Miguel Tuligtic, Ixtacamaxtitlan, Puebla & 2009 & Minera Gavilan S.A de C.V, Almaden Minerals & $\mathrm{Cu} \mathrm{Au} \mathrm{Ag}$ & \\
\hline $\begin{array}{l}\text { Municipio de Huehuetlán El Grande, Estado de } \\
\text { Puebla }\end{array}$ & 2009 & Minera Autlán S.A.B de C.V., Australian Minerals Group & $\mathrm{Mg}$ & \\
\hline Municipio Ensenada, Baja California & 2010 & $\begin{array}{l}\text { Grupo Mexico SA de CV, Southern Copper Corporation, } \\
\text { Minera México S.A de C.V. }\end{array}$ & $\mathrm{Cu} \mathrm{Mg} \mathrm{Au} \mathrm{Ag}$ & \\
\hline $\begin{array}{l}\text { Xochicalco, Mpios Miacatlán y Temixco, Estado de } \\
\text { Morelos }\end{array}$ & 2012 & $\begin{array}{l}\text { Esperanza Resources Corporation, Esperanza Silver México S.A } \\
\text { de C.V, Álamos Gold Inc. }\end{array}$ & $\mathrm{Au} \mathrm{Ag}$ & \\
\hline Estados de Colima y Jalisco & 2012 & $\begin{array}{l}\text { Ternium, Arcelor Mittal Corp, Consorcio Minero Benito Juárez } \\
\text { Peña Colorada, S.A. de C.V. }\end{array}$ & $\mathrm{Fe}$ & \\
\hline Tetela de Ocampo, Sierra Norte, Estado de Puebla & 2012 & Grupo Frisco, Minera Frisco S.A, Minera Espejeras S.A de C.V & $\mathrm{Au} \mathrm{Ag}$ & \\
\hline Santiago de Zautla, Sierra Norte, Estado de Puebla & 2012 & Jinduicheng Molybdenum Group Co., Ltd, JDC Minerals S.A. & Mo & \\
\hline Zacualpán, Colima & 2013 & Gabfer S.A de C.V & $\mathrm{Cu} \mathrm{Mg} \mathrm{Au} \mathrm{Ag}$ & \\
\hline Municipio Tlatlauquitepec, Estado de Puebla & 2013 & Minera Autlán S.A.B de C.V. & $\mathrm{Au}$ & \\
\hline Alto Lucero, Veracruz & ND & Gold Group & $\mathrm{Au}$ & \\
\hline Municipio de Ocampo, Estado de Coahuila & 1980 & Minera La Encantada S.A. de C.V., First Majestic Silver Corp & $\mathrm{Ag} \mathrm{Pb} \mathrm{Zn}$ & \\
\hline Real de Catorce, San Luis Potosí & 2008 & Ferro Gusa Carajás & $\mathrm{Ag}$ & \\
\hline San Antonio, Baja California Sur & 2010 & Argonaut Gold Corp., Minera Pitalla S.A de C.V & $\mathrm{Au}$ & \\
\hline Municipio de Tlahualilo, Estado Durango & 2011 & Minera Excellon de México S.A. DE C.V., Excellon Resources Inc. & $\mathrm{Ag} \mathrm{Pb} \mathrm{Zn}$ & \\
\hline Tequequitlán, Cuautitlán de García Barragán, Jalisc & 2013 & GanBo International Mining Co., Ltd. & Fe Mg & \\
\hline $\begin{array}{l}\text { Comunidad Salaverna, Municipio de Mazapil, } \\
\text { Zacatecas }\end{array}$ & 2013 & Minera Tayahua S.A. de C.V., Minera Frisco S.A & $\mathrm{Cu}$ & \\
\hline Chalchihuites, Zacatecas & 2014 & First Majestic Silver Corp & $\mathrm{Fe} \mathrm{Au} \mathrm{Ag}$ & \\
\hline Bahía de Ulloa, Baja California Sur & 2014 & $\begin{array}{l}\text { Oddisey Marine Exploration, Exploraciones Océnicas S. de R.L. } \\
\text { de C.V. }\end{array}$ & $\begin{array}{l}\text { Fosfato } \\
\text { marino }\end{array}$ & \\
\hline Sierra de Samalayuca, Estado de Chihuahua & 2015 & Samalayuca Cobre S.A. de C.V. & $\mathrm{Cu} \mathrm{Au} \mathrm{Ag}$ & \\
\hline Mulatos, Sonora & ND & Minera de oro nacional SA de CV & $\mathrm{Au}$ & \\
\hline Cerca Nogales, Sonora & ND & Grupo Carso / Grupo Frisco & $\mathrm{Cu}$ & \\
\hline San Antonio de la Cal, Tolimán, Estado de Querétan & ND & US Antimony & $\mathrm{Sb}$ & \\
\hline \multicolumn{5}{|l|}{ EJOLT } \\
\hline Michoacán & ND & Grupo México, IMMSA, Southern Peru Copper Corp. & $\mathrm{Au} \mathrm{Ag} \mathrm{Pb} \mathrm{Zn}$ & \\
\hline Oaxaca & 2002 & Natividad & $\mathrm{Au} \mathrm{Ag}$ & \\
\hline Chihuahua & 2006 & MagSilver & $\mathrm{Au} \mathrm{Ag}$ & \\
\hline Hidalgo & ND & Minera Autlán & Mg & \\
\hline \multicolumn{5}{|l|}{ EJOLT y OCMAL } \\
\hline Cocula, Guerrero & 2007 & Media Luna / Teck Cominco & $\mathrm{Au}$ & \\
\hline Chicomuselo, Chiapas & 2008 & Blackfire Exploration & $\mathrm{Ba}$ & \\
\hline San José del Progreso, Ocotlán, Oaxaca & 2009 & Fortuna Silver Mines Inc, Compañía Minera Cuzcatlán S.A de C. & $\mathrm{Au} \mathrm{Ag}$ & \\
\hline Municipio de Catorce, San Luis Potosí & 2010 & First Majestic Silver Corp., Revolution Resources Corp. & $\mathrm{Ag}$ & \\
\hline Magdalena de Tetipac, Tlacolula, Oaxaca & 2013 & BRigus Gold Corp (Antes Linear Gold), Plata Real Minerals Inc & $\mathrm{Ag}$ & \\
\hline CSP, San Luis Potosí & 1996 & New Gold / Minera San Xavier & $\mathrm{Au} \mathrm{Ag}$ & \\
\hline Coahuila & 2006 & Grupo México & C & \\
\hline Cananea, Sonora & 2007 & Grupo México /Southern Peru Copper Corp & $\mathrm{AuCu}$ & \\
\hline Los Cardones, La Paz, Baja California Sur & 2007 & Vista Gold Corporation, Invecture Group S.A. de C.V. & $\mathrm{Au}$ & \\
\hline La Purísima, Guanajuato & 2008 & Great Panther Resourses Limited | Minera Mexicana el Rosario & $\mathrm{Ag}$ & \\
\hline Chihuahua & ND & Minefinders Corporation of Vancouver & $\mathrm{Au}$ & \\
\hline
\end{tabular}

Elaboración propia con datos de OCMAL y EJOLT 2017. 
Para este trabajo, se revisaron las bases de concesiones, se tomó solamente la información que correspondía a minería aurífera y/o argentífera con concesiones vigentes para dar datos de un tipo de minería que se sabe altamente contaminante, extensiva, de mayoría de tipo tajo cielo abierto con lixiviación con cianuro, que no añade valor agregado ni genera encadenamientos productivos, que se encuentra dentro de la clasificación de extractivista, y que mantiene conflictos y demandas nacionales e internacionales.De esta manera se puede indicar claramente que existe un $4 \%$ del territorio concesionado a empresas trasnacionales de metales preciosos como primer elemento.Se seleccionaron concesiones vigentes, con sustancia principal oro o plata, las cuales suman 14, 621 titulares de concesiones, de un total de 32,447; de las cuales 2,920 están canceladas y 11,701 están vigentes. El 45\% de las concesiones totales son de minería de metales preciosos, de las cuales el $20 \%$ están canceladas y el $80 \%$ vigentes. Esto significa que el $36 \%$ de las concesiones mineras totales del país están dadas para procesar metales preciosos y están vigentes ${ }^{4}$.

Es necesario hacer hincapié en que existe una superficie que cubre el $68 \%$ de las concesiones totales en el país en etapa de exploración, lo que quiere decir que aún no se determina qué tipo de minería va a realizarse porque está en consideración el tipo de metales a extraer. Esto deriva en que todavía no se asienta la actividad directa, y es muy probable que se desconozca por parte de las poblaciones que serán afectadas, cuál empresa está entrando en su territorio. Esta proporción de superficie equivale al total del Estado de Chihuahua con 24.7 millones de hectáreas sobre los casi 196 millones de hectáreas con las que cuenta el territorio nacional.

Pueblos y sitios que habían sido abandonados o descartados dentro de la planeación regional, con niveles altos de marginalidad, exclusión social, migración, etc., ahora son lugares donde se pretenden realizar los megaproyectos. Jamás se les hacía caso por sus "necesidades", pero cuando son tomados en cuenta solamente es para que den su firma de aceptación, no de rechazo, sino que se conste que se harán cambios para "su propio bien".

\footnotetext{
${ }^{4}$ Hay un total de 32,433 concesiones, de ellas, 11,701 son concesiones de oro (Au) y plata (Ag), lo que equivale a un $36 \%$ de concesiones de metales preciosos respecto a las totales.
} 
Tabla 2. Número de proyectos mineros en conflicto y tipo de población.

\begin{tabular}{|l|r|r|r|}
\hline \multirow{2}{*}{$\begin{array}{l}\text { Empresas } \\
\text { mineras }\end{array}$} & \multirow{2}{*}{ Total } & \multicolumn{2}{|c|}{ Tipo de población } \\
\cline { 3 - 4 } & & Indígena & No Indígena \\
\hline Extranjera & 16 & 7 & 9 \\
Mixta & 16 & 10 & 6 \\
Nacional & 9 & 3 & 6 \\
Total & 41 & 20 & 21 \\
\hline
\end{tabular}

Elaboración propia con base en OCMAL y EJOLT 2017

Al no haber registro de la Secretaría de Economía y de la Dirección de Minas, del número de proyectos totales de capital nacional, trabajamos con los datos con que contamos que nos arrojaron que hayregistrados 32 de 41 proyectos mineros con capital extranjero en conflicto (según OCMAL y EJOLT), lo que complementando con la información anterior sobre el registro de 935 proyectos con capital extranjero, faltarían 903 proyectos que aún no se manifiestan como conflictos, es decir, tenemos una movilización y conocimiento de solamente el 3.4\% de los proyectos totales en el país (con capital extranjero o mixto), faltando además aquellos con capital nacional que desconocemos (solamente tenemos la cifra de 9 conflictos de empresas con capital nacional arrojada por los observatorios).

En otro orden, si bien la Ley Minera no cancela concesiones por haber incurrido en (posibles) faltas al medio ambiente o por (posibles) daños a poblaciones donde se albergará la minería, si ocurre por terminación de su vigencia, desistimiento del titular, por sustitución de nuevos títulos de concesión, por resolución judicial, o como medida de sanción. La sanción de cancelación se da por infringir las siguientes normas: Explotar minerales o sustancias no permitidas en la Ley Minera, no tener actividades, no cubrir los derechos sobre minería, no cumplir pagos ni presentar informes, no sujetarse a especificidades de los minerales que retiran (carbón por ejemplo), no tener los permisos especiales de autoridades en casos como Áreas Naturales Protegidas (ANP), Zonas marítimas, etc., no tener concesiones colindantes, omitir hallazgos de hidrocarburos y perder la capacidad para ser titular de concesiones.

Por tanto, no importa el daño que puedan y lleguen a hacer las mineras, la concesión no se retira por impacto directo o indirecto ni afectaciones de tipo ambiental, social, económico, cultural a la población local. El recurso de cancelación por sanción únicamente comprende cuestiones que incurran en faltas administrativas y burocráticas. 


\section{MOVILIZACIÓN DE LOS AFECTADOS POR LA MINERÍA}

Las poblaciones que se enfrentan a los proyectos mineros tienen información sobre los aspectos por los que se verían afectados por la entrada de las mineras, por lo que movilizan discursos y acciones de defensa en diferentes frentes. Es tan fuerte la carga simbólica, tangible e intangible de lo que se vería vulnerado, que las redes que se tienden hacia las poblaciones en resistencia (y re-existencia) coinciden en que no solo debe atacarse a la minería, sino que debe lucharse contra cualquier otro proyecto ${ }^{5}$ que atente e interrumpa la autogestión para la vida en sus territorios.

Ahora bien, entre las redes que se identifican frente a la imposición de proyectos mega-mineros y ante el despojo de su capacidad de autogestión destacan dos actores que han ayudado a establecer estrategias de largo alcance: la Red de Afectados por la Minería (REMA) y el Movimiento Mesoamericano contra el Modelo Extractivo Minero (M4). En sus comunicados, destacan algunos elementos que han podido conseguir con las comunidades afectadas: a) Territorios libres de minería, b) Comunicación entre comunidades y c) Documentación de daños y acciones por parte de las empresas. Este tejido nos habla de las mezclas de distintas poblaciones de afectados en pugna entre los que aceptan y los que se oponen a los proyectos mineros (campesinos, indígenas, ejidatarios, avecindados, comuneros, afectados, implicados...); sin embargo, el gran logro que se ha obtenido de muchas de estas luchas en red, ha sido la suspensión de proyectos mineros, aunque, como ya se dijo, no su cancelación. Muchos proyectos se encuentran en fase de suspensión, pero no por ello significa que se cancelan o se retiran las concesiones, éstas continúan hasta que la empresa determina oportuno continuar (ver Tabla 2). Por mencionar un ejemplo, el caso de Tetela de Ocampo, Puebla, nos refiere a una lucha que derivó en la suspensión de los permisos de funcionamiento y obra de la Minera Frisco, pero la concesión actualmente se encuentra vigente, y la etapa de la actividad minera según el registro que tiene la Secretaría de Economía, es de exploración, por lo tanto, la empresa continúa en actividades "normales", solo tiene "en pausa" el trámite administrativo 6 .

\footnotetext{
${ }^{5}$ También conocidos como los han definido las comunidades de la Sierra Norte de Puebla: "Proyectos de Muerte".

${ }^{6}$ Información de la página de la Secretaría de Economía, Proyectos mineros. 
Tabla 3. Tipos de procesos y fases de los proyectos mineros

\begin{tabular}{|l|l||l|l|}
\hline \multicolumn{1}{|c|}{ CONCESIÓN } & \multicolumn{1}{|c|}{$\begin{array}{c}\text { PERMISO DE } \\
\text { FUNCIONAMIENTO, } \\
\text { OBRA }\end{array}$} & $\begin{array}{c}\text { ETAPA DE LA } \\
\text { ACTIVIDAD MINERA } \\
(\text { SE) }\end{array}$ & $\begin{array}{l}\text { FASE TÉCNICA DE LA } \\
\text { ACTIVIDAD MINERA }\end{array}$ \\
\hline $\begin{array}{l}\text { Vigente } \\
\text { Cancelada } \\
\text { Suspendida }\end{array}$ & $\begin{array}{l}\text { Otorgado } \\
\text { Cancelado } \\
\text { Suspendido }\end{array}$ & $\begin{array}{l}\text { Exploración } \\
\text { Desarrollo } \\
\text { Producción } \\
\text { Postergación }\end{array}$ & $\begin{array}{l}\text { Prospección } \\
\text { Exploración } \\
\text { Explotación } \\
\text { Beneficio } \\
\text { Abandono }\end{array}$ \\
\hline
\end{tabular}

Elaboración propia con base en Dirección General de Desarrollo Minero y Ley minera

Por ello, cuando hablamos de los conflictos socioambientales que se generan alrededor de la imposición de los proyectos mineros, se vuelve necesario detectar qué es lo que se protege, lo que se intenta preservar por parte de la población afectada. Es así que, como ya hemos planteado anteriormente, las movilizaciones se dan en general a favor de la libre determinación sobre sus territorios, que no dependen forzosamente de los límites administrativos ni del tipo de propiedad física de áreas determinadas. Deleuze y Guattari (1998) refieren al territorio como una noción más amplia “en dimensiones que van de lo físico a lo mental, de lo social a lo psicológico" (Ramírez y López, 2015: 127).

No obstante, las dimensiones del territorio caben en una perspectiva más amplia, incluso política del espacio. Mencionan Blanca Ramírez y Liliana López (2015) que el territorio es "la superficie terrestre sujeta a procesos de posesión, soberanía, gestión, dominio, administración, control, resistencia, utilización, explotación, aprovechamiento, apropiación, apego y arraigo", es aquello entonces que se posee en conjunto, en comunidad, como un proyecto en común.

La manera en cómo se realiza esta apropiación es mediante prácticas, en la cotidianidad, dándole un sentido ontológico con bases epistémicas propias. En el momento en que se generan diversos procesos sociales diferenciados, este territorio genera territorialidades propias de los sujetos y actores que en él se desenvuelven, en distintas escalas tanto temporales como espaciales. Parte de estas territorialidades son la forma en que se relaciona la población con los elementos físicos concretos, tales como los ríos, montañas o barrancos y relaciones con otros sujetos como los muros, barricadas y trincheras (Ramírez y López, 2015: 148). Aunque no siempre se trata de objetos físicos, también hay elementos intangibles, de corte simbólico. Para Ramírez y López (Ibíd.:133) este entramado de territorialidades, es la suma de sentimientos de pertenencia que se construyen a partir del habitar, reconociendo el sentido de pertenencia y al arraigo. Este territorio no se ve tanto en 
términos de "propiedad" (aunque se reconoce la propiedad colectiva y los "comunes"), sino, de apropiación efectiva mediante prácticas culturales, agrícolas, ecológicas, económicas, rituales, etcétera (Escobar, 2014: 90).

Sin importar de quién es la propiedad que se esté pisando, la población local se ha relacionado con el territorio más allá de los límites "privados" o de la propiedad social (ejidos y comunidades agrarias). En espacios afectados por la minería, se reconocen los pasos de herradura, las veredas para el pastoreo, las áreas de recolección de frutos silvestres, tunas, garambullos, cactáceas varias, lugares por donde la gente pasa, los vive y se apropia, son suyos sin ser su propiedad, son sus "territorios". No obstante, cuesta más trabajo defender lo que no se habita, como los sitios con afectaciones históricas por una minería realizada desde épocas coloniales, y más tarde en el porfiriato o en pleno siglo XX, comprenden espacios que han sido abandonados, deteriorados, descuidados continuamente, lo que ha obstaculizado una plena autogestión, no el desarrollo de capacidades para tomar decisiones de corte territorial. Esto ha derivado en un proceso de cierta desterritorialización, el cual, como menciona Escobar (2014: 128), se expande por visiones diversas a partir de la ruptura de los paradigmas de la modernidad en la última década del siglo XX y del inicio del modelo de globalización neoliberal en el mundo. Desterritorialización significa "la tendencia creciente de los Estados, en el contexto del capitalismo global, de encontrar y fomentar el desarraigo de la gente y de las cosas, con grandes consecuencias sociales, psicológicas y políticas” (Ibídem: 152). Esta des-apropiación no se refiere a que el espacio físico deja de tener importancia, sino que los vínculos entre un grupo social y su espacio se debilitan. En los casos afectados por la minería, existe un trabajo continuo por parte de los gobiernos para "preparar el terreno" para la llegada de las empresas mineras. Lo que sucede como contraparte es que se están retomando las riendas de los territorios por los pueblos y comunidades, echando marcha atrás a procesos de desterritorialización, reapropiándose de sus propias prácticas, reconociendo en ellas sus saberes y conocimientos, re-territorializando.

Esta suerte de retorno marca una nueva etapa, donde se reconfiguran nuevos territorios, construcciones sociales que proceden de patrimonios ambientales y culturales, como dimensiones vinculadas por razones evidentes con los conocimientos técnicos así como con las instituciones (en el amplio sentido), los valores sociales y las representaciones. Es así que el territorio comprende patrimonios, a partir de la importancia que adquiere por su dimensión simbólica, por medio de la identificación con una cosmovisión, mitologías y prácticas rituales particulares que han sido construidos en el tiempo. 
Es muy importante destacar cómo estos patrimonios bioculturales (Boege, 2013) son movilizados a partir de la manera en que percibe la población este tipo de amenazas en su territorio, y los relaciona al enfrentarlos directamente con su experiencia vivida, cotidianidad, manera de relacionarse con el entorno en que habitan. Las formas en cómo se lleva esta relación, se pueden describir como menciona Escobar (2014: 16) dentro de un sentipensar con el territorio, que implica pensar desde el corazón y desde la mente, o co-razonar, es la forma en que las comunidades territorializadas han aprendido el arte de vivir. Sentipensar con los territorios, culturas y conocimientos de sus pueblos — con sus ontologías-, más que con los conocimientos des-contextualizados que subyacen a las nociones de "desarrollo", “crecimiento" y "economía”.

Escobar (2014: 59), menciona que las ontologías relacionales con frecuencia involucran perspectivas territoriales y comunales, como en el caso de comunidades indígenas, afrodescendientes y campesinas. En estas ontologías, los territorios son espacios-tiempos vitales de interrelación con el mundo natural. Aquí radica la importancia de una perspectiva ontológica sobre los conflictos ambientales. Por ejemplo, en sitios donde han sido continuamente despojados, la manera de sobrevivir ante la incertidumbre y abandono, también forma parte de estas ontologías y perspectivas territoriales. Es una dinámica que se ha presentado y podemos decir interiorizado, desde la primera explotación minera en la época colonial. Son más de cinco siglos de colonialidad y neocolonialidad que seguimos llevando dentro, no importa si es una población netamente indígena o si es "mestiza", los procesos de dominación continúan. La minería puede decirse en esta perspectiva, se ha mantenido dentro de los parámetros de dominación desde que llegó por primera vez y continúa hasta nuestros días con su estructura jerárquica y la implantación no solamente de un sistema productivo depredador, sino desde un rasero social y cultural que impone y deshecha cualquier manifestación que no sea la que pretende consolidar el capitalismo neocolonial.

Frente a esa estructura de dominación, todo proceso contra-hegemónico se construye acumulando saberes. La sabiduría de los pueblos lleva al conocimiento de los límites, riesgos, daños, de las maneras de superar obstáculos o peligros. Es desde el patrimonio cultural, social y natural, visto así, como patrimonio, y con ello distinguir que está en riesgo, junto con las relaciones y prácticas que tienen las poblaciones con su entorno, y por eso mismo se movilizan para conseguir su protección y se despliega el derecho por su autogestión. 


\section{EL PATRIMONIO BIOCULTURAL Y LAS NUEVAS FORMAS DE DEFENSA DEL TERRITORIO}

Desde el momento en que la ley determina que la minería es prioritaria, se rechaza todo planteamiento alternativo en los marcos legales, por lo que el actor que decide el rumbo del territorio es la empresa, y no solamente durante la actividad extractiva, sino por hasta cien años ${ }^{7}$. Esto constituye un despojo del derecho básico de gestionar los territorios en libertad y no bajo las cláusulas operativas de una empresa. Es así que el conflicto que se abre es tanto sociocultural, como socioambiental. No es necesario en este sentido que sean exclusivamente los pueblos indígenas los que sufren desplazamientos e impactos. Las actividades mineras han derivado, desde épocas coloniales hasta nuestros días, en dinámicas que han afectado a los sitios donde se alojan, especialmente las regiones rurales, dejando pasivos ambientales sin atención, tejido social fisurado y poblaciones en conflicto, vulnerables y despojadas de sus derechos de decidir sobre su territorio.

Ahora bien, ¿qué es lo que contiene y ocurre en el territorio para que exista un interés por defenderlo? Cuando se hace referencia a lo cultural y a lo ambiental, se habla de las relaciones que se mantienen con un espacio determinado, ese intercambio entre las poblaciones y su entorno. Pero hemos venido diciendo que el espacio territorial es definido por los pueblos y comunidades como patrimonio biocultural, que como lo define Eckart Boege (2008: 13), se refiere a los "recursos naturales bióticos intervenidos en distintos gradientes de intensidad por el manejo diferenciado y el uso de los recursos naturales según patrones culturales, los agroecosistemas tradicionales, la diversidad biológica domesticada con sus respectivos recursos fitogenéticos desarrollados y/o adaptados localmente". Estas actividades se desarrollan alrededor de prácticas productivas (praxis) organizadas bajo un repertorio de conocimientos tradicionales (corpus) y relacionando la interpretación de la naturaleza con ese quehacer, el sistema simbólico en relación con el sistema de creencias (cosmos) ligados a los rituales y mitos de origen (Boege, Ibíd.).

Es así que ambiente y cultura forman desde nuestro punto de vista, un todo, sin separación y perfilan epistemologías distintas, que no tienen que ser las hegemónicamente

${ }^{7}$ La Ley Minera en su artículo 15 menciona: Las concesiones mineras tendrán una duración de cincuenta años, contados a partir de la fecha de su inscripción en el Registro Público de Minería y se prorrogarán por igual término si sus titulares no incurrieron en las causales de cancelación previstas en la presente Ley y lo solicitan dentro de los cinco años previos al término de su vigencia. DOF 26 de junio de 1992. Párrafo reformado DOF 28-04-2005. 
impuestas. La cultura, así vista deriva de prácticas que se mantienen en regiones no indígenas o mestizas, que también forman parte de este mosaico cultural en el que estamos inmersos y de alguna manera se relacionan en el momento de proponer esfuerzos conjuntos para poder luchar y plantear nuevos mundos posibles en procesos de autoreconocimiento para reinventarse, a la vez que se espejean con los demás movimientos u organizaciones.

De manera práctica, las ONG's, estudiantes, asesores y otros tipos de actores interactúan con poblaciones tanto mestizas como indígenas a la hora de elaborar sus estrategias, y no significa la imposición de uno o de otro lado, es una práctica interdisciplinaria e intercultural, en donde lo que se valora es el reconocimiento de un territorio y las territorialidades derivadas de las prácticas bioculturales que ahí se llevan a cabo. De estos intercambios, se ha detectado una la influencia de una gran diversidad de movimientos e incluso la compartición de estrategias o acompañamientos en las luchas.

La idea que entendemos ha sido básica entre los movimientos socio-ambientales es que han ampliado la visión de quiénes son los encargados del resguardo de los bienes naturales, que rompe con una visión restringida y reduccionista producto de cierto relativismo cultural, como en el caso de la llamada ética del cuidado, cuando se designa a las mujeres porque son las que mejor lo hacen, evadiendo responsabilidades que también pueden realizar los hombres y sobre todo, cargando de más de trabajo a las mujeres. Para replantear las estrategias, hay que distinguir que las poblaciones no solo están resistiendo, sino reexistiendo, en tanto practican "formas de re-elaborar la vida auto-conociéndose como sujetos de la historia interpelada en su horizonte de colonialidad como lado oscuro de la modernidad occidental y reafirmando lo propio sin que esto genere extrañeza, revalorando lo que nos pertenece desde una perspectiva crítica frente a todo aquello que ha propiciado la renuncia y el auto-desconocimiento" (Albán, 2009: 70).

El re-existir es un renacer en medio de tensiones políticas por la renovación de las relaciones jerárquicas entre los grupos que componen la sociedad, por lo que implicare-definir y resignificar la vida en condiciones de dignidad y autodeterminación, enfrentando la biopolítica que controla, domina y mercantiliza a los sujetos y la naturaleza. Esto plantea ir mucho más allá de la relación entre culturas y apunta a cuestionar seriamente las desigualdades de las estructuras de poder, las inequidades de todo tipo, la racialización y la marginalización de grupos étnicos, el adultocentrismo decisorio, el relegamiento y sometimiento de la mujer en el contexto de las estructuras patriarcales y la negación de diversas alternativas en lo sexual, lo político y lo religioso (Albán, 2009: 85-86). 
Con la mega-minería es palpable este dominio y sometimiento en sitios que históricamente han sido despojados de prácticas que no sean dentro de los parámetros mineros, dejándolos sin alternativas para decidir sus propias vidas. Siguiendo a Adolfo Albán (Ibídem: 455), la re-existencia forma parte de los dispositivos que las comunidades crean y desarrollan para inventarse cotidianamente la vida y poder de esta manera confrontar la realidad establecida por el proyecto hegemónico que desde la colonia hasta nuestros días ha inferiorizado, silenciado y visibilizado negativamente. La re-existencia apunta a descentrar las lógicas establecidas para buscar en las profundidades de las culturas, las claves de formas organizativas, de producción, alimentarias, rituales y estéticas que permitan dignificar la vida y re-inventarla para permanecer transformándose: “¿Qué nos vamos a inventar hoy para seguir viviendo?" ". Si además, recuperamos el planteamiento de Guillermo Bonfil (1987), de que el "México profundo" está formado por una gran diversidad de pueblos, comunidades y sectores sociales que construyen la mayoría de la población del país. Lo que los une y los distingue del resto de la sociedad mexicana es que son grupos portadores de maneras de entender el mundo y organizar la vida que tienen su origen en la civilización mesoamericana, forjado aquí a lo largo de un dilatado y complejo proceso histórico sentidos de pertenencia, percepción de identidad, resguardo de lo que es suyo. A partir de lo anteriormente dicho, analizamos un caso específico, quizá paradigmático.

\section{EL CASO DEL CERRO DE SAN PEDRO, SAN LUIS POTOSÍ (MÉXICO)}

El Cerro de San Pedro es un caso central, referente, de las luchas contra la mega minería, así como para retomar la autogestión de su territorio en esta fase de (supuesto) cierre de la mina. Hay que empezar diciendo que este poblado ha sido marcado históricamente por la minería, pues desde el descubrimiento de sus minas de oro y plata en 1592, ha sido objeto de abandonos y despoblamientos continuos en función de los intentos por recuperar el metal sepultado. Así, desde esa historia colonial en el presente ha sufrido un nuevo embate de desterritorialización producto del rompimiento de las relaciones entre sus pobladores, y debilitamientos internos graves. Esto ha sido parte de una estrategia por parte del Estado para "preparar el terreno" para las inversiones extranjeras.

\footnotetext{
${ }^{8}$ Planteamiento del líder comunitario, cooperativo y sindical Héctor Daniel Useche Berón "Pájaro", asesinado en 1986 en el Municipio de Bugalagrande en el centro del Valle del Cauca, Colombia, cuando le preguntaron por el concepto de re-existencia, según Albán (2009: 455).
} 
Desde la década de 1990 hubo una serie de acciones por parte de los gobiernos en turno para desalojar el poblado de Cerro de San Pedro, primero se canceló el transporte público al pueblo, se retiró la escuela y el archivo histórico, incluso se intentó suprimir la cabecera municipal para pasarla al poblado de Portezuelo, pero no prosperó la iniciativa. De esta forma se encontrarían las mejores condiciones para las inversiones mineras, pues se podría explotar el cerro con poca o nula población habitando el sitio. Bajo este panorama desolador en términos demográficos y de viabilidad económica, comenzó en 1995 en esta zona la exploración por parte de la compañía minera canadiense Metallica Resources $\operatorname{Inc}^{9}$ y su subsidiaria mexicana Minera San Xavier (MSX). En palabras de la empresa: "El proyecto de Cerro de San Pedro sería desarrollado utilizando minado convencional de Tajo a Cielo Abierto y el proceso metalúrgico de Lixiviación en Montones con soluciones de baja concentración de cianuro y recuperación de metales preciosos por medio del sistema conocido como Merrill-Crowe" (MIA, 1997).

A grandes rasgos, el conflicto puede resumirse en las siguientes etapas de confrontación. La primera, es cuando se logra que se interrumpa el proceso de permisos en 1998, lo que significó un obstáculo para la empresa, aunque un año después consiguió tener una autorización condicionada. En una segunda etapa que arranca en 2004 los opositores a la mina logran que la justicia declare nula la resolución, descartando cualquier permiso y autorización dado. Sin embargo, en una tercera etapa, la MSX vuelve a realizar el trámite y se le otorga un nuevo permiso en 2006; y la empresa consigue instalarse por completo y obtiene su primer lingote de oro y plata (doré). En 2009, abren una cuarta etapa los opositores al lograr de nuevo por medios judiciales, que se declare ilegal el permiso dado en 2006, ordenando el cierre permanente el mes de noviembre del mismo año. No obstante, al llegar la fecha, la instancia encargada del cierre anuncia que únicamente hará inspección de rutina sin ningún otro efecto. Es en este sentido que hablamos con anterioridad de un cierre, no de las minas, sino de los canales legales para oponerse a este modelo de muerte.

Y también, cuando hablamos del sentipensar, nos referimos a la forma en que las comunidades por medio de sus prácticas, de sus territorialidades, han aprendido el arte de vivir; aunque, los pobladores de Cerro de San Pedro más que aprender el arte de vivir, ejercieron el oficio de sobrevivir, lo que no quiere decir que todo haya sido sufrimiento, porque su sobrevivencia vino acompañada del aprendizaje que trae formar una identidad y una manera de vida, incierta, pero que formó tradiciones y que formaron una manera de

${ }^{9}$ Desde el año 2000 pasó a manos de la empresa canadiense Newgold Inc. 
sentipensar su territorio, de una manera de relacionarse con su tierra, sus entorno, sus habitantes otros, sus plantas y sus cerros. Se pensaron en y desde la incertidumbre, se sintieron en abandono, pero como diría Arturo Escobar (2014: 58) la montaña fue vista "como un ser sintiente", tratada de una muy otra forma.

La quinta etapa parte del año 2014, cuando la MSX emitió comunicados donde anunciaba que estaba proyectando el cierre de sus operaciones para 2016. Ante estas declaraciones, los grupos opositores pidieron la divulgación pública del plan oficial del cierre de la empresa, y con ello que la población interesada y afectada pudiera incidir directamente en los criterios de la clausura; hasta la fecha, inicios de 2018, no han conseguido una respuesta oficial, y solamente les han proporcionado una presentación en línea de un programa básico en la página de la Cámara Minera de México $\left(\right.$ CAMIMEX) ${ }^{10}$, donde se destaca este proyecto como minería “con responsabilidad social”. Dicho programa ha sido revisado y analizado por diversos miembros y simpatizantes de la oposición a la MSX, y se ha dictaminado que los planes expuestos carecen de fundamentos técnicos, legales, sociales y culturales. La empresa incurre incluso en la apropiación demagógica de propuestas que fueron generadas hace tiempo por la población opositora ${ }^{11}$.

Sobre un territorio fragmentado, donde se ejerció desde el poder un proceso de desterritorialización, ahora los pobladores trabajan en la reconstrucción, la reconfiguración, la re-territorialización, recurriendo incluso a aquellos rituales que se habían perdido y los retoman desde un nuevo contexto resignificándolos para re-apropiarse de lo que era suyo, reexistiendo.

\section{A MANERA DE REFLEXIONES ABIERTAS QUE NO FINALES}

En el ejemplo del pueblo Cerro de San Pedro, la re-existencia, se muestra como la capacidad de tomar elementos tradicionales y reinventarse, cuando ya se estaban perdiendo, para darse un nuevo sentido, volver a estructurarse y tomar las riendas que les fueron arrebatadas. La re-existencia es también una postura crítica, política, de vida al replantear lo existente y hacerlo trascender, no como una simple suma de proyectos aislados que

\footnotetext{
${ }^{10}$ Información de la página de la CAMIMEX y NEW GOLD - MSX, (2015)
}

Disponible en: http://www.camimex.org.mx/files/6914/4112/8074/2-2.pdf

${ }^{11}$ Información obtenida de entrevista a Mario Martínez Ramos, habitante de Cerro de San Pedro y miembro del patronato pro defensa, mayo 2016, y de la publicación "El huachichil", órgano informativo de la casa cultural de Cerro de San Pedro, 2016. 
contemplen turismo, vivienda, tierra, sino en marcar una pauta para inventarse una forma de vida autónoma, digna, armónica. Las prácticas y cotidianidades desde la minería, también forman territorio. La sobrevivencia y el reconocimiento de cómo ha sido esta la manera de vida a la cual no se quiere volver, forma parte del re-existir.

El colonialismo-neocolonial es la clave para la desterritorialización, incluyendo la de los saberes, como aquello que toma forma en las dicotomías campo-ciudad, modernidadatraso. La desterritorialización se acompaña de violencia y despojo, eliminación y criminalización del otro, invasión. Esta desterritorialización, se vuelve factible cuando los territorios están fisurados, en contraste con aquellos sitios que mantienen relaciones más directamente y durante más tiempo (con mayor continuidad) con su territorio (ancestral, comunal).

Retomar la memoria colectiva como el elemento que une a los poblados de la región, incluso que deja de lado los posicionamientos en cuanto a llevar o no a cabo la minería, lo importante radica en no volver a perder la capacidad de autogestión, hacer trabajo común, donde se forma un sujeto. Hacer consciente aquello que se había vivido, pero no se había reconocido con carga simbólica, ontológica, sobre su propia constelación de saberes. Esta relación viene de una lógica y una tradición histórica que ha sido dada por la minería, pero donde esa minería, ya no sea vista como un destino inevitable, sino como el punto de partida para reconocerse desde una historia no oficial.

El tendido de redes sociales fue amplio y no pertenece a una condición de exclusividad cultural. La participación se expresó en términos "difusos", desde diferentes actores que ayudaron al reconocimiento deseado. Estas redes hicieron recordar que las luchas parten en ocasiones de información que es desconocida por la población local, por lo que se necesita una vinculación de conocimientos relacionados con temas como minería, agua, fracking, cuencas, especies en peligro de extinción, etc., que la misma población acaba por ampliar desde su experiencia.

Asistimos a nuevas formas para identificar los elementos de defensa, al cobrar fuerza elementos como el paisaje, los itinerarios, rutas, geopatrimonios (en el sentido del patrimonio geológico). El Cerro se torna un elemento vivo y a la vez no vivo, pero no por ello, menos importante. Lo no vivo son los minerales que tienen además de ser una carga simbólica cultural (son parte del escudo de San Luis Potosí), sus propiedades metálicas únicas como el contenido de plomo y la aleación plata/oro poco vista en la mineralización del país. Lo vivo 
como parte del sistema ambiental, captación de agua, regulación de vientos, diversidad de flora y fauna.

Urge destacar que esta creciente oleada de minería se vincula con otros megaproyectos (también llamados de muerte). Asimismo, habla de lo que las propias comunidades pretenden preservar y que identifican que existe un riesgo de desaparición ante la actividad minera y los megaproyectos, se trata entonces, de proyectos de vida frente a los proyectos de muerte, es una lucha cósmica, civilizatoria y contra el capitalismo neocolonial. El derecho a las territorialidades se basa más que en denunciar, legitimar las formas de vida, la reconstrucción y la reinvención de modos de habitar y co-habitar como parte de unos "muy otros" derechos colectivos, comunes, los de una otra economía como modos de producción de vida, de luchas y alianzas.

Finalmente, y no por ello al final, pervive la necesidad del reencuentro entre movimientos que han declarado e impuesto territorios libres de mega-proyectos de minería a cielo abierto con las luchas de aquellos otros movimientos que han surgido de los impactos de la minería y que per-viven, re-existiendo en la incertidumbre.

\section{BIBLIOGRAFÍA}

Acosta, Alberto (2009), La maldición de la abundancia, Abya-Yala, Quito.

Acosta Alberto (2012), "Extractivismo y neoextractivismo: Dos caras de la misma maldición”, en Lang, Miriam y Dunia Mokrani (comps.), Más allá del desarrollo, ABYA YALA, Fundación Rosa Luxemburgo, pp. 83-118.

Albán Achinte, Adolfo (2013), "Pedagogías de la re-existencia. Artistas indígenas y afrocolombianos", en Walsh, Catherine (editora), Pedagogías decoloniales. Prácticas insurgentes de resisitir, (re) existir y (re) vivir. Tomo I. Serie Pensamiento DecolonialAbyaYala, Quito.

Alimonda, Héctor (2011), "La colonialidad de la naturaleza. Una aproximación a la Ecología Política Latinoamericana" en H. Alimonda, (coord.), La colonización de la naturaleza. Ecología política y minería en América Latina, CLACSO, Buenos Aires.

Boege, Eckart (2013), "La minería industrial en territorios bioculturales de los pueblos indígenas. El despojo de los indígenas de sus territorios en el siglo XXI", Revista electrónica Rebelión, http://www.rebelion.org/noticias/2013/6/169166.pdf

Boege, Eckart (2015), "Patrimonio biocultural y los derechos culturales de los pueblos indígenas, comunidades locales y equiparables". Mimeo; Ciudad de México. 
Bonfil B., Guillermo (1987), México profundo. Una civilización negada, CIESAS/SEP, Foro 2000, Ciudad de México.

Escobar, Arturo (2014), Sentipensar con la tierra. Nuevas lecturas sobre desarrollo, territorio y diferencia, Ediciones UNAULA, Medellín.

Espinosa, Gisela y Patricia Castañeda (2015), “Género, seguridad alimentaria y cambio climático" en Dante Ariel Ayala, Dolores Camacho, Yolanda Castañeda y Artemisa López (coord.), Crisis civilizatoria y sus dilemas tecnológicos y socioambientales. Respuestas desde los actores sociales, México, AMER, UAM-X, UMSNH, UNACh, pp. 29-54.

Metals Economics Group (2012). Exploración minera. Tendencias de exploración mundial, Centro de Estudios del Cobre y Minería, Canadá.

Núñez Rodríguez, Violeta; Couturier Bañuelos, Patricia; y Concheiro Bórquez, Luciano (2013), “Las mineras en México: ¿desarrollo para quién?” en F. Novelo, El retorno del desarrollo. UAM-X; México, D.F.

Ramírez Velázquez, Blanca Rebeca y Liliana López Levi (2015), Espacio, paisaje, región, territorio y lugar: la diversidad en el pensamiento contemporáneo, Instituto de Geografía, UNAM, UAM-Xochimilco, Ciudad de México.

Svampa, Maristella (2012), "Extractivismo neodesarrollista y movimientos sociales. ¿Un giro ecoterritorial hacia nuevas alternativas?” en Miriam Langy Dunia Mokrani (comps.), Más allá del desarrollo, ABYA YALA, Fundación Rosa Luxemburgo, pp. 185-218. 